\title{
Vertical meridian asymmetry in spatial resolution: Visual and attentional factors
}

\author{
CIGDEM P. TALGAR and MARISA CARRASCO \\ New York University, New York, New York
}

\begin{abstract}
We investigated whether spatial resolution would be the same in the lower and upper halves of the vertical meridian (VM) of our visual field and whether attention would affect them differentially. It has been reported that (1) attending to the target's location improves performance in a texture segregation task when the observer's spatial resolution is too low (peripheral locations) but impairs it when resolution is already too high (central locations) for the task. This finding indicates an enhanced spatial resolution at the attended location (Yeshurun \& Carrasco, 1998, 2000), (2) observers' contrast sensitivity is higher in the lower than in the upper VM, a phenomenon known as vertical meridian asymmetry (VMA), an asymmetry determined by visual rather than by attentional factors (Carrasco, Talgar, \& Cameron, 2001). In the present texture segregation task, performance was assessed under neutral- and peripheralcue conditions. Transient covert attention was systematically manipulated by using a peripheral cue that indicated the target's location and its onset. Observers reported the interval containing a target patch appearing at one of a number of eccentricitiesin a large texture pattern along the VM. We found that (1) performance peaked at farther eccentricities in the lower than in the upper visual VM, indicating that resolution was higher in the lower half, and (2) the peripheral cue affected performance along the VM uniformly, indicating that the degree of enhanced resolution brought about by transientattention was constant along the VM. Thus, we conclude that the VMA for spatial resolution is determined by visual, not transient covert attentional, constraints.
\end{abstract}

Spatial covert attention is the selective processing of visual information at a given location in the absence of eye movements to that location (e.g., Posner, 1980). Several authors have characterized two components of covert attention and have found that it can be allocated to the target location either voluntarily, according to the observer's goals (sustained attention), or involuntarily, in a reflexive manner (transient attention; e.g., Cheal \& Lyon, 1992; Jonides, 1981; Nakayama \& Mackeben, 1989). A number of studies have explored the way in which covert attention affects early vision (e.g., Foley \& Schwartz, 1998; Lee, Itti, Koch, \& Braun, 1999; Lee, Koch, \& Braun, 1997; Lu \& Dosher, 1998; Morgan, Ward, \& Castet, 1998). In particular, our lab has demonstrated that transient attention enhances both contrast sensitivity (Carrasco, Penpeci-Talgar, \& Eckstein, 2000) and spatial resolution (Carrasco \& Yeshurun, 1998;

Grant NSF/BCS-9910734 and a Cattell fellowship to M.C. and a Katzell Summer Fellowship to C.P.T. supported this research, which was presented at the meeting of the Visual Sciences Society (May, 2001) and at the European Conference in Visual Perception (August, 2001). We thank Leslie Cameron, Fani Loula, Barbara Montagna, Damian Stanley, Joanna Tai, Patrick Williams, and Yaffa Yeshurun for their valuable comments on an earlier version of this manuscript. Correspondence should be addressed to M. Carrasco, Department of Psychology, New York University, 6 Washington Pl. 8th fl., New York, NY 10003 (e-mail: marisa. carrasco@nyu.edu).
Yeshurun \& Carrasco, 1998, 1999, 2000), two basic dimensions of vision.

In the studies mentioned above, performance has been assessed independently of the target's location in the visual field. However, performance across the visual field is not homogeneous. A horizontal-vertical anisotropy (HVA) denotes better performance on the horizontal than on the vertical meridian (VM; e.g., Carrasco, Talgar, \& Cameron, 2001; Rijsdijk, Kroon, \& Van der Wilt, 1980; Rovamo \& Virsu, 1979). Furthermore, performance is superior in the lower than in the upper visual field (e.g., Berardi \& Fiorentini, 1991; Edgar \& Smith, 1990; Nazir, 1992; Rubin, Nakayama, \& Shapley, 1996). We have recently reported that this asymmetry is more pronounced along the VM-a phenomenon known as vertical meridian asymmetry (VMA); specifically, contrast sensitivity is superior in the lower half of the VM (Carrasco et al., 2001). Establishment of the presence of a VMA in spatial resolution would indicate that the heterogeneity of the visual system constrains performance in all visual tasks since both contrast sensitivity and spatial resolution underlie the processing of all visual stimuli and that vertical asymmetry may play a prominent role in tasks that require high resolution. Furthermore, such an asymmetry across the VM would pose a confounding variable for studies presenting stimuli at different locations in the visual field. 
(a)

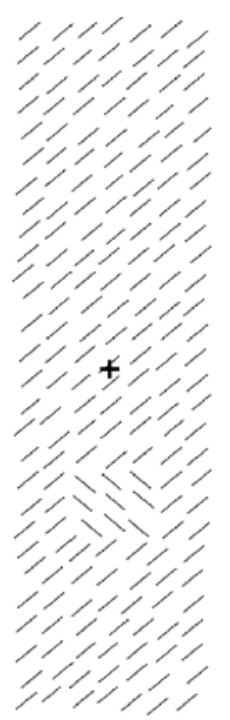

(b)

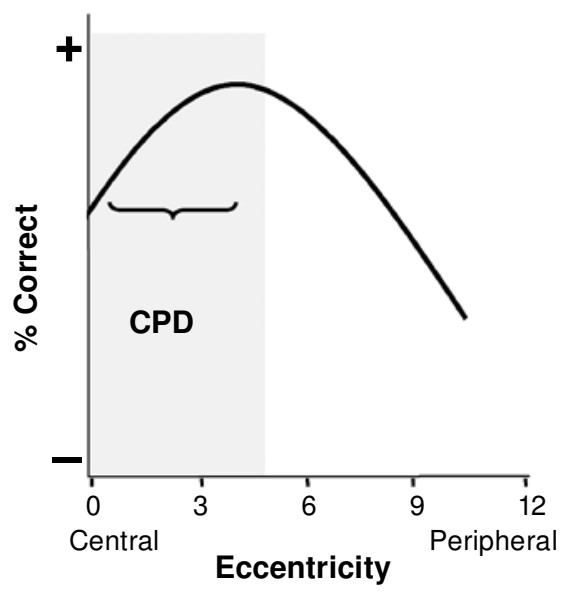

Figure 1. (a) An example of the display used in the texture segregation. The target appeared equally often at one of 17 different locations along the background texture. (b) A graph of the performance function in a texture segregation task. Performance peaks at midperipheral locations and drops as the target appears at more central or farther peripheral locations (after Gurnsey, Pearson, \& Day, 1996). The highlighted section depicts the central performance drop (CPD).

Some recent studies have attributed the inhomogeneity in performance across the visual field to attention. For instance, an HVA reported in a letter identification task (Mackeben, 1999) and a vertical asymmetry reported in a Snellen acuity task (Altpeter, Mackeben, \& TrauzettelKlosinski, 2000) have been attributed to a sustained attentional mechanism. Similarly, superior performance in the lower visual field in search and tracking tasks has been attributed to higher attentional resolution in the lower visual field (He, Cavanagh, \& Intrilligator, 1996). As has been established in visual search research (Carrasco, Evert, Chang, \& Katz, 1995; Carrasco \& Frieder, 1997; Carrasco, McLean, Katz, \& Frieder, 1998; Geisler \& Chou, 1995; Verghese \& Nakayama, 1994), before an attentional explanation is invoked, it is important to rule out the more parsimonious explanation that performance is determined by visual constraints. However, studies that have attributed the inhomogeneity in performance across the visual field to attention have neither ruled out the visual factors underlying these heterogeneities nor explicitly manipulated attention.

To evaluate the attentional explanation of performance inhomogeneities across the visual field, we have systematically characterized the effect of transient attention on contrast sensitivity (Carrasco et al., 2001). Both the HVA and the VMA become more pronounced as spatial frequency, eccentricity, and the number of distractors increase in a wide range of tasks - orientation discrimination, detection, and localization - with different stimulus orientations and luminance levels, and with both monocular and binocular viewing conditions). In contrast to those who have attributed performance fields to attention, we have found that although transient attention improves overall performance, it does not interact with target location. Furthermore, attention shifts the psychometric function for contrast sensitivity to lower contrasts similarly at all locations in the visual field (Cameron, Tai, \& Carrasco, 2001). Because in these two studies on contrast sensitivity similar performance fields emerged in conditions that did or did not invoke transient attention, we have concluded that performance fields are determined by visual, not transient attentional, constraints.

To directly assess the issue of attentional resolution's being higher in the lower hemifield (He, Cavanagh, \& Intrilligator, 1996), in the present study, we explicitly manipulated attention and measured how it would affect spatial resolution across the VM. To test this, we used a texture segregation task and evaluated whether directing observers' transient attention to the target location influenced performance differentially in the upper and lower VM. Early visual processing is thought to be responsible for texture segregation, the ability to segregate a target from its surround (see Figure 1a). In this task, performance is constrained by spatial resolution (Gurnsey, Pearson, \& Day, 1996; Joffe \& Scialfa, 1995; Kehrer, 1989; Morikawa, 2000; Yeshurun \& Carrasco, 1998, 2000). Unlike in most static visual tasks (e.g., Carrasco \& Frieder, 1997; DeValois \& DeValois, 1988; Graham, 1989), in the texture segregation task, performance is not optimal at fovea. As is illustrated in Figure 1b, in such a texture segregation task, performance peaks at midperipheral locations and drops as the target appears at more central or farther peripheral locations (Gurnsey et al., 1996; Joffe \& Scialfa, 1995; Kehrer, 1989; Morikawa, 2000; Pearson \& Gurnsey, 1992; Yeshurun \& Carrasco, 1998, 2000). Psychophysical and physi- 
ological evidence indicate that we process visual stimuli by means of parallel spatial filters, each tuned to a band of spatial frequencies (DeValois \& DeValois, 1988; Graham, 1989). Particularly relevant to the present study is the characteristic central performance drop (CPD) in the texture segregation task. Such a drop is considered to reflect a mismatch between the average size of spatial filters responsible for the spatial resolution in that region of the visual field and the scale of the texture. With retinal eccentricity, the average size of the filters decreases and consequently resolution increases. Whereas these filters are too small in the fovea (i.e., resolution is too high), they are too large in the periphery (i.e., resolution is too low; Gurnsey et al., 1996; Joffe \& Scialfa, 1995; Kehrer, 1989; Morikawa, 2000).

A number of studies have shown that attention enhances spatial resolution (Carrasco \& Yeshurun, 1998; Reynolds \& Desimone, 1999; Yeshurun \& Carrasco, 1998, 1999, 2000). For instance, a peripheral, transient cue improves performance more for peripheral than for central targets in a visual search task, implying that attention can reduce resolution differences between the fovea and the periphery (Carrasco \& Yeshurun, 1998). Furthermore, the findings that precuing the target location with a peripheral cue improves performance in gap resolution tasks and that this benefit becomes more pronounced as target eccentricity increases provide further support for the spatial resolution hypothesis (Carrasco, Williams, \& Yeshurun, 2002; Yeshurun \& Carrasco, 1999).

In a critical test of this hypothesis, Yeshurun and Carrasco (1998) explored the effects of attention on the basic texture segregation task (described above) in which performance is diminished by heightened resolution (see Figure 1). Given that performance is already worse at the fovea because its spatial filters are too small and have too high a resolution for the scale of the texture (Gurnsey et al., 1996; Morikawa, 2000),,$^{1}$ further increasing resolution at foveal locations should lead to a more pronounced drop in performance. Attention helped performance at peripheral locations, but hindered performance at central locations. ${ }^{2}$ The mere fact that attention affected performance is noteworthy. Texture segregation based on orientation differences is considered an early preattentive task (see, e.g., Joffe \& Scialfa, 1995; Julesz, 1981; Northdurft, 1991). Note that these results are inconsistent with all models of attention, which predict that attention always helps and never hinders performance. Such impairment could be mediated only by an attentional mechanism that enhances resolution by effectively decreasing the average size of filters at the attended location. It has also been determined that by enlarging the scale of the texture, the peak of performance is shifted to farther eccentricities, consistent with the hypothesis that segregation of larger textures requires larger filters that are more abundant at farther eccentricities (Gurnsey et al., 1996). Consequently, for a bigger texture scale, attention hinders performance for a larger central region, resulting in a larger CPD (Yeshurun \& Carrasco, 1998).

An attractive feature of this texture segregation task is that it provides a link with known properties of the physiological substrate of vision (e.g., Gurnsey et al., 1996; Yeshu- run \& Carrasco, 2000). Physiological evidence indicates that responses capable of underlying segregation occur in cortical area V1 (e.g., Lamme, Van Dijk, \& Spekreijse, 1993; Purpura, Victor, \& Katz, 1994). Given that the texture segregation task is limited by spatial resolution, it provides an ideal paradigm for investigating whether spatial resolution also exhibits the VMA reported for contrast sensitivity (Carrasco et al., 2001). Furthermore, when attention is explicitly manipulated, the pattern of results can be explained only by an attentional mechanism that enhances spatial resolution (Yeshurun \& Carrasco, 1998). However, these authors tested the effect of attention on texture segregation only along the horizontal meridian. Whereas previous studies had averaged performance over all equally eccentric locations (Joffe \& Scialfa, 1995; Kehrer, 1989; Pearson \& Gurnsey, 1992; Yeshurun \& Carrasco, 1998, 2000), our aim was to compare the upper and lower VM. We oriented the texture pattern along the VM and presented the target at varying eccentricities up to $12^{\circ}$ to investigate whether (1) performance in a texture segregation task differs along the VM, and (2) the effect of transient attention differs throughout the VM. We hypothesized that (1) spatial resolution would be superior in the lower half of the VM, and (2) transient attention would increase spatial resolution consistently across the VM.

We illustrate these hypotheses in Figure 2. Because the performance function in the texture segregation task results from the extent of the match between the size of the spatial filters and the texture scale, the higher the spatial resolution, the more pronounced the CPD and the more eccentric the performance peak. Figure 2a shows a greater spatial resolution in the lower than in the upper half of the VM. If, at a given eccentricity, filters were smaller in the lower than in the upper half, the lower half would show a greater CPD and would peak at a farther eccentricity. Figure $2 b$ depicts the enhancement of spatial resolution throughout the VM as a result of transient attention. This is because a peripheral transient cue impaired performance at central locations and shifted the performance peak to a farther eccentricity along the horizontal meridian (Yeshurun \& Carrasco, 1998, 2000). Figure $2 \mathrm{c}$ portrays a consistent degree of attentional effect across the two halves of the VM. This effect is assessed by the point at which the attentional effect crosses over from an impairment to an improvement. If the lower half of the vertical meridian had a higher resolution, the attentional crossover point would be more eccentric in the lower than in the upper half of the vertical meridian. Therefore, the difference between the two graphs in Figure 2c would be predicted by spatial resolution alone-that is, without a differential effect of attention along the two VMs).

\section{EXPERIMENT}

\section{Method}

Observers. Fourteen New York University undergraduates participated for credit in a psychology course. All had normal or corrected-to-normal vision and were naive as to the purpose of the study.

Apparatus. An Apple G4 computer was used to display the stimuli on a gamma-corrected 21-in. Sony P51 monitor. Stimuli were pre- 

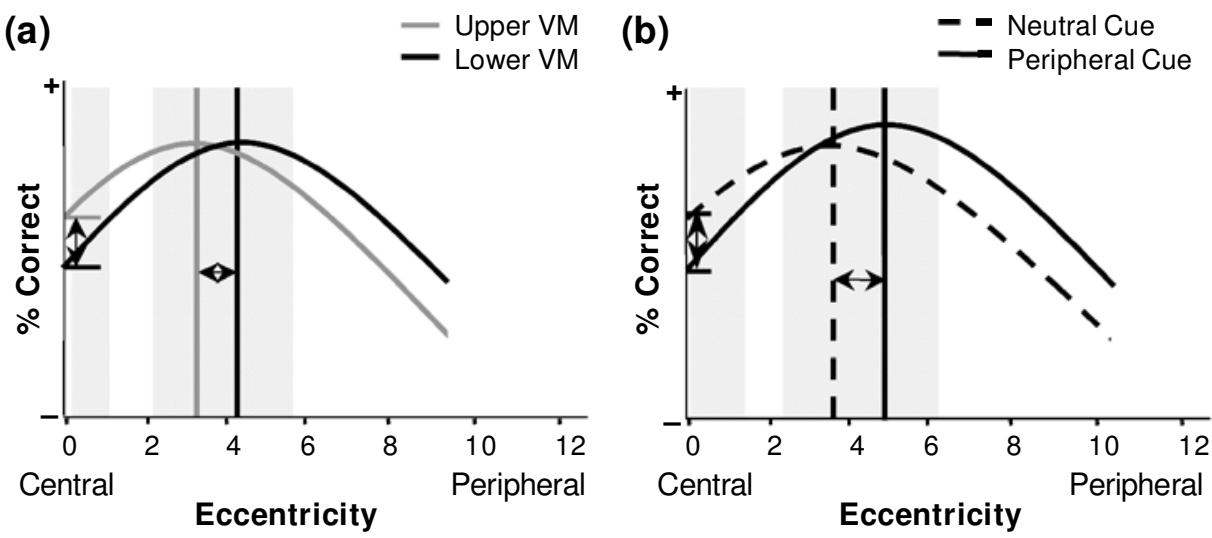

\section{(c) Upper Vertical Meridian}
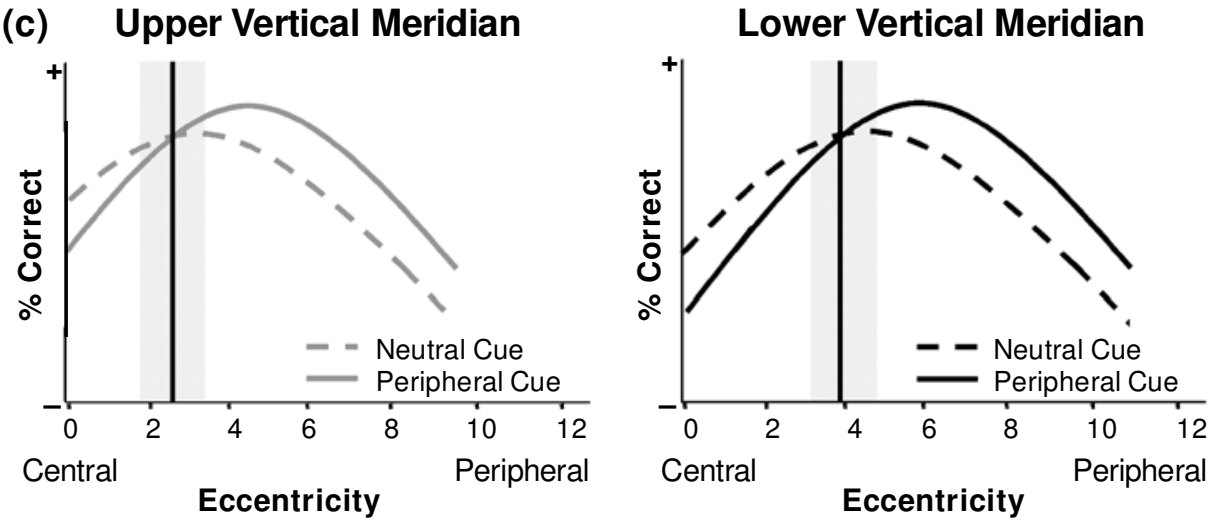

Figure 2. (a) Pattern of results that would reflect a greater spatial resolution in the lower than in the upper vertical meridian (VM). The lower half would show a greater central performance drop (CPD) and would peak at a farther eccentricity than would the upper half. The two horizontal lines depict the greater CPD, and the two vertical lines depict the more eccentric peak for the lower VM (see text). (b) The overall attention effect across the entire VM. The two horizontal lines depict the greater CPD, and the two vertical lines depict the more eccentric peak for the peripheral cue condition compared with the neutral cue condition (see text). (c) A consistent degree of attentional effect across the VM. The line in each graph identifies the point where the attention impairment crosses over to an advantage. The left graph shows this point for the upper VM, and the right graph shows it for the lower VM. The higher the resolution, the more eccentric the crossover point from an attentional impairment to an advantage should be (see text).

sented by the PsyScope software (Cohen, MacWhinney, Flatt, \& Provost, 1993).

Stimuli and Design. At a viewing distance of $57 \mathrm{~cm}$, the texture pattern subtended $14^{\circ} \times 5^{\circ}$ of visual angle above and below the center of the display (Figure 3 ). It consisted of 287 lines oriented at $45^{\circ}$ or $135^{\circ}$ (41 rows $\times 7$ columns), in which a target texture $(3 \times 3$ lines oriented orthogonal to the background subtending $1.5^{\circ} \times 2^{\circ}$ of visual angle) was embedded in the background texture.

Procedure. Figure 3 depicts the sequence of events within one trial. The observers viewed the display binocularly. In each trial, there were two intervals, each of which began with a fixation point at the center of the screen, followed by one of two possible cues presented for $70 \mathrm{msec}$. A peripheral precue indicated the target location. To prevent forward masking, this precue, a green horizontal bar $\left(0.9^{\circ} \times\right.$ $0.5^{\circ}$ ), appeared $0.3^{\circ}$ beyond the target's distant edge from the fixation point. The timing between the precue and display onsets $(110 \mathrm{msec})$ maximized the attentional effect (Nakayama \& Mackeben, 1989) and precluded eye movements (Mayfrank, Kimmig, \& Fischer, 1987). The peripheral precue appeared in $50 \%$ of the trials. Given that it appeared in both intervals and always indicated the target location in the interval that contained the target ( $100 \%$ valid), the precue did not associate a higher probability with one of the responses, and the observers could not rely on its presence to reach a decision. In the other $50 \%$ of the trials, a "neutral" cue, a green vertical line $\left(28^{\circ} \times 0.3^{\circ}\right)$, appeared along each side of the texture, indicating that the target was equally likely to appear in any of 17 possible locations ( 1 central location, 8 above and 8 below, ranging from $0.7^{\circ}$ to $11.75^{\circ}$ eccentricity). After an interstimulus interval of $40 \mathrm{msec}$, the texture pattern appeared for an average of $40 \mathrm{msec}$. The presentation time for the texture ranged from 30 to $50 \mathrm{msec}$ so that each observer would perform at about $80 \%$ correct. Following the display, a mask (containing crosses, made of $45^{\circ}$ and $135^{\circ}$ lines), spanning the display, appeared for $200 \mathrm{msec}$. Following the observer's response, a "+" or "-" sign indicated a correct or incorrect response, respectively. There were three blocks of 300 trials each for a total of 900 experimental trials (50 datapoints per target location and cue combination) per observer. On half of the trials, the target appeared in the first of two intervals. The observers were asked to indicate the interval that contained the target by pressing one of two keys.

\section{Results}

We performed a 2 (peripheral vs. neutral cue) $\times 2$ (upper vs. lower VM) $\times 9$ (eccentricity) repeated mea- 


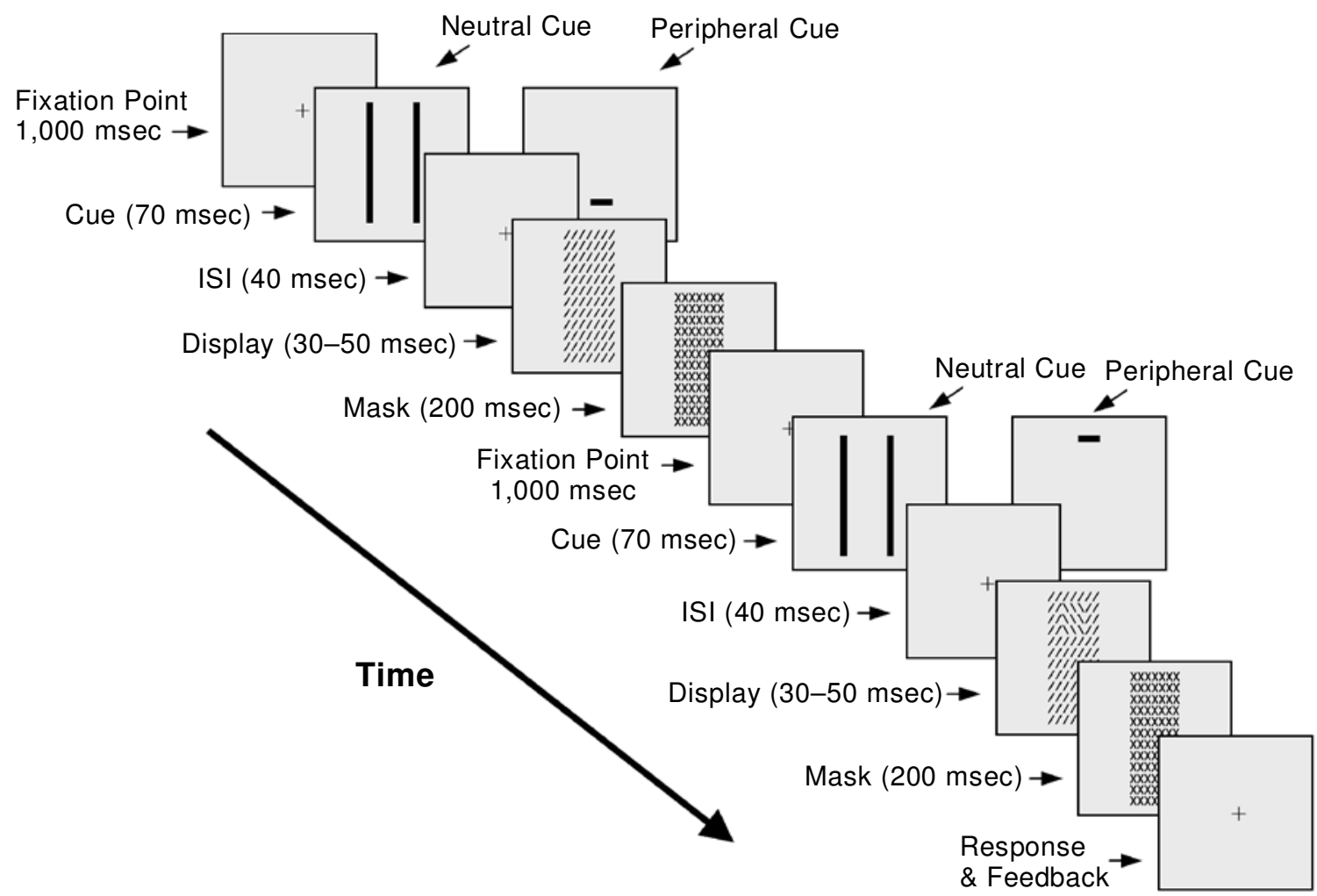

Figure 3. Representation of a trial sequence. In half the trials, the target was preceded by a peripheral cue (a small line located above the location of the target), and the rest of the trials were preceded by a neutral cue (two vertical lines spanning the entire range of the texture pattern on both sides; see text).

sures analysis of variance (ANOVA) to evaluate the three predictions presented in the introduction. ${ }^{3}$

Resolution differences between the upper and lower vertical meridian. In Figure $4 a$, the neutral cue performance functions are compared for the two halves of the VM. The quadratic fits for the upper and lower halves had $R^{2}$ values of .95 and .75 , respectively. The ANOVA revealed a significant hemifield $\times$ eccentricity interaction $[F(8,104)=2.85, p<.01]$ : The CPD was more pronounced and the performance peaked at a farther $\left(6.4^{\circ}\right)$ eccentricity for the lower than for the upper half $\left(5^{\circ}\right)$. These results confirm our predictions (see Figure 2a).

Cuing Effect. Figure 4b, shows the overall effect of the peripheral and neutral cue on texture segregation collapsed over the two halves of the VM. The $R^{2}$ value for the quadratic fits for the neutral and peripheral cues were .92 and .97 , respectively. The ANOVA revealed a significant cue type $\times$ eccentricity interaction $[F(8,104)=2.17, p<.05]$ : The cuing of target location impaired performance at central locations but enhanced performance at farther eccentricities. Furthermore, performance peaked at a farther eccentricity for the peripheral than for the neutral cue $\left(6.5^{\circ}\right.$ vs. $5.5^{\circ}$ eccentricity). These results confirm our predictions (Figure 2b) and are consistent with Yeshurun and Carrasco $(1998,2000)$.

Interaction between cuing and location in the VM. In Figure 4c, the effect of the neutral and peripheral cues is plotted for the lower (right graph) and upper (left graph) halves of the VM. Consistent with our predictions (Figure $2 \mathrm{c}$ ), the ANOVA showed that neither the cue type $\times$ hemifield interaction $[F(1,13)=2.44, p>.1]$ nor the threeway interaction $[F(8,104)=1.03, p>.1]$ was significant. This pattern of results indicates that the magnitude of the cuing effect did not differ on the basis of whether the target appeared in the upper or lower VM. Furthermore, the crossover point at which the attentional effect switched from a benefit to an impairment occurred $1.3^{\circ}$ farther in eccentricity for the lower $\left(4^{\circ}\right)$ than for the upper $\left(2.7^{\circ}\right)$ half of the VM.

Figure 5a illustrates the extent of the difference in spatial resolution between the lower and upper halves of the VM in the neutral condition. To equate performance for both halves at eccentricities near fixation and to maximize the overlap of both functions, we shifted the function for the lower half $1^{\circ}$ toward the left (so that its starting point would be -1$)$. The resulting graph indicates that after performance peaks, it declines in a more pronounced fashion in the upper than in the lower half of the VM. In Figure 5b, a similar effect is depicted for the peripheral cue; equating performance at the near eccentricities by displacing the lower curve toward the left (so that its starting point would be -1 ) resulted in a perfect overlap of both functions. This illustrates that the peripheral cue increased resolution in both halves of the VM in such a way that the resulting per- 

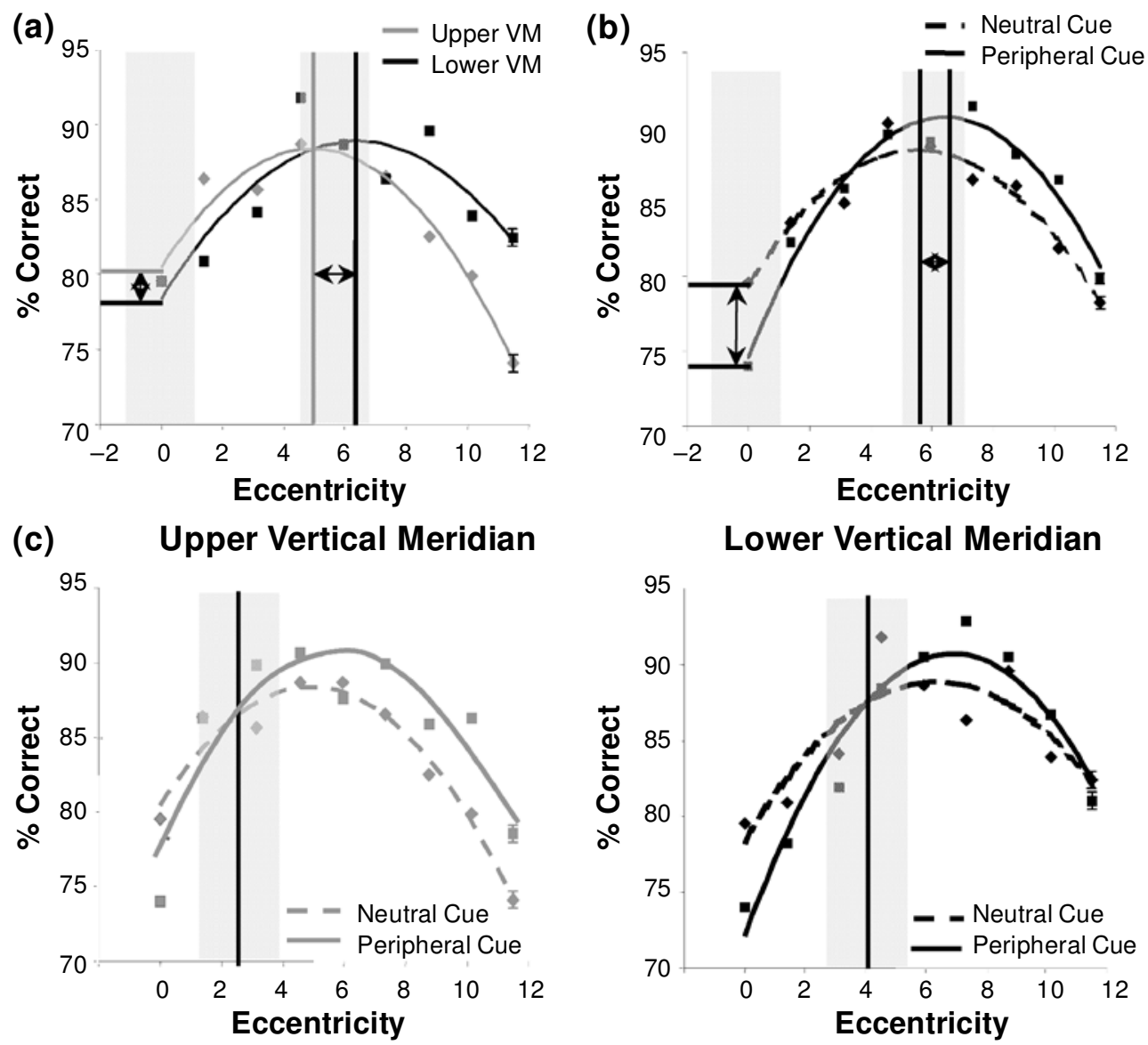

Figure 4. (a) Comparison of performance as a function of eccentricity and the half of the meridian in which the target appeared for the neutral condition. The two horizontal lines depict the greater central performance drop (CPD), and the two vertical lines point out the more eccentric peak for the lower vertical meridian (VM). (b) Graph of the overall effect of the peripheral cue and neutral cue on texture segregation as a function of eccentricity irrespective of which half of the meridian the target appeared. The two horizontal lines depict the greater CPD, and the two vertical lines depict the more eccentric peak for the peripheral cue condition compared with the neutral cue condition. (c) The individual effects of the peripheral cue and neutral cue for the lower (left graph) and upper half (right graph) of the VM. The line in each graph identifies the point at which the attention impairment crosses over to an advantage for the upper (left graph) and lower (right graph) halves of the VM. Error bars give $\pm S E$ for each condition.

formance difference was tantamount to $1^{\circ}$ eccentricity. These two graphs, together with the lack of a significant three-way interaction, indicate that the magnitude of the cuing effect did not differ significantly along the VM.

\section{DISCUSSION}

We used a texture segregation task in which performance decreases at central locations because the filters' sizes at those locations are too small for the scale of the texture and consequently, the spatial resolution is too high (Gurnsey et al., 1996; Joffe \& Scialfa, 1995; Kehrer, 1989; Morikawa, 2000). By presenting the texture pattern along the VM, this task enabled us to assess how spatial resolution differs along this meridian. To our knowledge, this is the first study to provide psychophysical evidence for higher spa- tial resolution in the lower than in the upper VM. Furthermore, the extent to which resolution declines is greater in the upper than in the lower VM. Both of these findings are consistent with the finding that contrast sensitivity differs along the VM (Carrasco et al., 2001).

There is an apparent inconsistency in the literature regarding the existence and the degree of an asymmetry between the upper and lower visual fields. A closer look at these studies reveals that the terms VMA and upper-lower visual field differences have been used inconsistently and, in some cases, interchangeably. Here we note that the existence of the VMA is not equivalent to an overall difference between all of the possible locations in the upper and lower visual fields. On the one hand, the reported advantage of the lower visual field in a variety of psychophysicaltasks (see, e.g., Edgar \& Smith, 1990; Nazir, 1992; Previc, 1990; 
(a) Neutral Cue

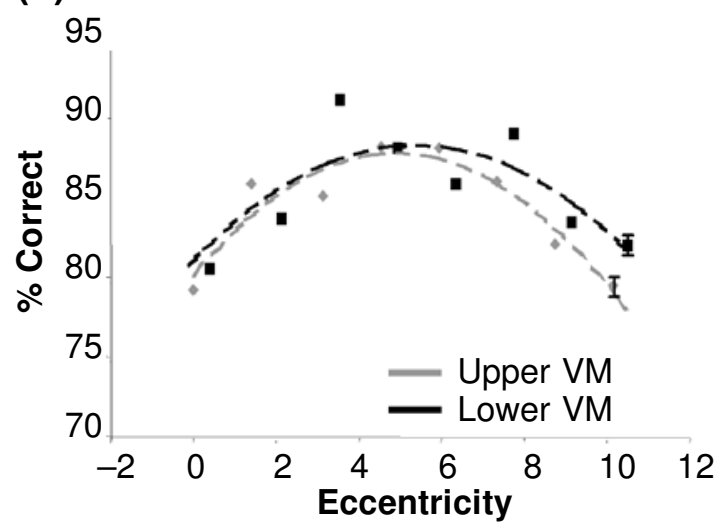

(b) Peripheral Cue

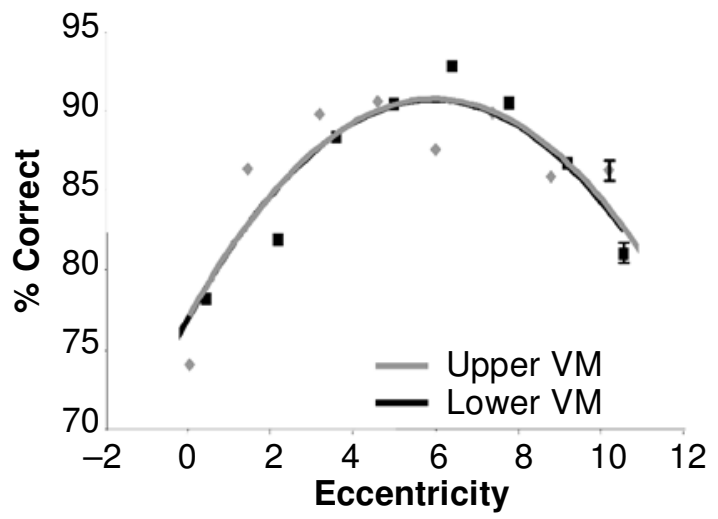

Figure 5. (a) Illustration of the extent of the difference in spatial resolution between the lower and upper vertical meridian (VM) in the neutral condition. To equate performance for both halves at eccentricities near fixation and to maximize the overlap of both functions, we shifted the function for the lower half $1^{\circ}$ toward the left (so that its starting point would be -1 ). (b) The results of equating performance at the near eccentricities for the peripheral cue by displacing the lower curve toward the left (so that its starting point would be -1 ), resulting in a perfect overlap of both functions (see text). Error bars give $\pm S E$ for each condition.

Rubin et al., 1996) might have been primarily driven by differences along the VM and generalized to the entire hemifield. On the other hand, some studies have reported a lack of a significant difference between the upper and lower visual fields (e.g., Carrasco et al., 1995; Carrasco \& Frieder, 1997; Carrasco et al., 1998). In those cases, averaging across several locations is likely to have diminished or even obscured a real difference along the VM. This explanation could also be responsible for the small lower field advantage reported for a similar texture segregation task with both simple and complex texture channels (Graham, Rico, Offen, \& Scott, 1999). However, when the target appears along only the $\mathrm{VM}\left(10^{\circ}\right.$ eccentricity $)$ in a similar segregation task, the asymmetry is more pronounced (Hofmann \& Hallett, 1993). Thus, these inconsistent findings could be explained by the specific location where the target appeared in the visual fields; when they are restricted to the $\mathrm{VM}$, the asymmetry is more pronounced.

Possible neural correlates of the VMA for spatial resolution include the greater cone and ganglion cell densities in the lower than in the upper VM (Perry \& Cowey, 1984, 1985), and the fact that slightly more area is devoted to the inferior than to the superior visual field in LGN (Connolly \& Van Essen, 1984) and cortical area V1 (Tootell, Switkes, Silverman, \& Hamilton, 1988; Van Essen, Newsome, \& Maunsell, 1984). To our knowledge, no neural correlates have been reported that would account for our finding that the extent to which spatial resolution declines is more pronounced in the upper than in the lower VM.

It is reasonable to assume that attentionalmodulation may reflect a combination of mechanisms such as reduction of external noise-attention diminishes spatial uncertainty and facilitates distracter exclusion (Lu, Liu, \& Dosher, 2000; Palmer, 1994; Shiu \& Pashler, 1994, 1995; Solomon, Lavie, \& Morgan, 1997; Sperling \& Dosher, 1986)—and signal enhancement-attention strengthens the stimulus' representation (e.g., Carrasco et al., 2000; Carrasco et al., 2002; Lu \& Dosher, 1998; Müller et al., 1998). By presenting the texture display along the VM, we found that attention impairs performance at central locations while enhancing it at far eccentricities. These results are consistent with those of Yeshurun and Carrasco (1998, 2000), who used a texture presented along the horizontal meridian. Note that although the attentional benefit at peripheral locations is consistent with many mechanisms, the attentional impairment at central locations can be explained only by enhanced spatial resolution.

The explanation that attention enhances spatial resolution also accounts for the finding that attention improves detection and discrimination performance in tasks that have been developed to assess acuity (e.g., Landolt square; Carrasco et al., 2002) and hyperacuity (vernier offset; Yeshurun \& Carrasco, 1999). Indeed, in a recent review, Reynolds and Desimone (1999) considered that these psychophysical studies lend support to the neurophysiological studies showing that attention increases spatial resolution by contracting the neurons' receptive field around the attended stimulus (Desimone \& Duncan, 1995; Luck et al., 1996; Moran \& Desimone, 1985; Reynolds \& Desimone, 1999).

Previous studies have attributed the HVA (Altpeter et al., 2000; Mackeben, 1999) and VA (Altpeter et al., 2000) to sustained attention. Given that the transient component of attention is considered to operate at an earlier stage of visual processing than the sustained component (e.g., Nakayama \& Mackeben, 1989), before an attentional explanation is invoked, it is important to rule out the likely possibility - and more parsimonious explanation-that visual constraints also underlie the performance fields. In the present study, we show that when covert attention is explicitly manipulated via a peripheral precue, the extent to which transient attention increases resolution along the VM does not dif- 
fer systematically. That is, although we do report that the lower VM has a higher resolution than the upper VM, transient attention does not affect resolution more in one or the other half. ${ }^{4}$

The present results lend support to the idea that transient attention increases resolution to a similar degree at different locations in the visual field. These results are consistent with a recent study on contrast sensitivity in which by systematically manipulating both transient attention and visual factors, we found that (1) contrast sensitivity is superior in the lower VM, (2) as eccentricity increases, the VMA in contrast sensitivity is more pronounced, and (3) the attentional effect did not interact with the location along the $\mathrm{VM}$ at which the target was presented. We concluded that the performance fields for contrast sensitivity are determined by visual, rather than by attentional, constraints (Carrasco et al., 2001). Likewise, in this study, we found that (1) spatial resolution is greater in the lower than in the upper $\mathrm{VM},(2)$ the rate of decline for spatial resolution as a function of eccentricity is more pronounced in the upper half, and (3) the attentional effect did not interact with the location along the VM at which the target was presented. Thus, we conclude that the VMA for spatial resolution is determined by visual, not transient attentional, constraints.

The resolution differences we report along the VM could reflect ecological constraints. In most circumstances, there is more visual information in the lower than in the upper half of our visual field. Previc (1990), for instance, has proposed that the upper and lower visual fields are functionally specialized for far and near vision, respectively, so that stimuli are processed more efficiently in the lower than in the upper visual field. Indeed, it is possible that the inferior visual field is more important for survival. Under most viewing conditions, the sky would take up a majority of the superior visual fields, at least in primates living outside the natural forest (Tootell et al., 1998). The existence of VMA has several implications. For instance, regarding experimental design and analysis, the VMA suggests that either the stimuli should be presented in areas of the visual field with similar characteristics so that the results can be generalized, or performance should be analyzed for different locations independently. In addition, the VMA suggests ergonomic and human factors applications (e.g., when one is designing an instrument panel, critical information should not be presented at the upper VM since the visual system's efficiency to process information at this location is compromised by its low contrast sensitivity and spatial resolution).

The present results are consistent with the findings that covert attention increases performance in two basic dimensions of early vision-namely, spatial resolution (Morgan et al., 1998; Tsal \& Shalev, 1996; Yeshurun \& Carrasco, 1998, 1999, 2000) and contrast sensitivity (e.g., Cameron et al., 2001; Carrasco et al., 2000; Carrasco et al., 2001; Foley \& Schwartz, 1998; Lee et al.,1999; Lee et al.,1997; Lu \& Dosher, 1998; Solomon et al., 1997). Furthermore, they conclusively show that regardless of the inhomogeneity of the visual field - that is, HVA and VMA - the attentional effect is constant throughout the VM.

\section{REFERENCES}

Altpeter, E., Mackeben, M., \& Trauzettel-Klosinski, S. (2000). The importance of sustained attention for patients with maculopathies. Vision Research, 40, 1539-1547.

Berardi, N., \& Fiorentini, A. (1991). Visual field asymmetries in pattern discrimination: A sign of asymmetry in cortical visual field representation? Vision Research, 37, 831-836.

Cameron, E. L., TaI, J. C., \& Carrasco, M. (2001). Covert attention affects the psychometric function of contrast sensitivity. Vision Research, 42, 949-967.

Carrasco, M., Evert, D. L., Chang, I., \& Katz, S. M. (1995). The eccentricity effect: Target eccentricity affects performance on conjunction searches. Perception \& Psychophysics, 57, 1241-1261.

Carrasco, M., \& Frieder, K. S. (1997). Cortical magnification neutralizes the eccentricity effect in visual search. Vision Research, 37, 63-82.

CArrasco, M., \& McElree, B. (2001). Covert attention speeds the accrual of visual information. Proceedings of the National Academy of Sciences, 98, 5363-5367.

Carrasco, M., McLean, T. L., Katz, S. M., \& Frieder, K. S. (1998). Feature asymmetries in visual search: Effects of display duration, target eccentricity, orientation and spatial frequency. Vision Research, 38, 347-374.

Carrasco, M., Penpeci-Talgar, C., \& Eckstein, M. (2000). Spatial attention increases contrast sensitivity across the CSF: Support for signal enhancement. Vision Research, 40, 1203-1215.

Carrasco, M., Talgar, C., \& Cameron, L. (2001). Characterizing visual performance fields: Effects of transient covert attention, spatial frequency, eccentricity, task, and set size. Spatial Vision, 14.

Carrasco, M., Williams, P., \& Yeshurun, Y. (2002). Covert attention increases spatial resolution with or without masks: Support for signal enhancement. Journal of Vision, 2, 467-479.

Carrasco, M., \& Yeshurun, Y. (1998). The contribution of covert attention to the set-size and eccentricity effects in visual search. Journal of Experimental Psychology: Human Perception \& Performance, 24, 673-692.

Cheal, M. L., \& Lyon, D. R. (1992). Benefits from attention depend on the target type in location-precued discrimination. Acta Psychologica, 81, 243-267.

Cohen, J., MacWhinney, B., Flatt, M., \& Provost, J. (1993). PsyScope: An interactive graphic system for designing and controlling experiments in the psychology laboratory using Macintosh computers. Behavior Research Methods, Instruments, \& Computers, 25, 257-271.

Connolly, M., \& VAN Essen, D. (1984). The representation of the visual field in parvocellular and magnocellular layers of the lateral geniculate nucleus in the macaque monkey. Journal of Comparative Neurology, 266, 544-564.

Desimone, R, \& Duncan, J. (1995). Neural mechanisms of selective visual attention. Annual Review of Neuroscience, 18, 193-222.

DeValois, R. L., \& DeValois, K. K. (1988). Spatial vision. New York: Oxford University Press.

Edgar, G. K., \& Smith, A. T. (1990). Hemifield differences of perceived spatial frequency. Perception, 19, 759-766.

Foley, J. M., \& Schwartz, W. (1998). Spatial attention: Effect of position uncertainty and number of distracter patterns on the thresholdversus-contrast function for contrast discrimination. Journal of the Optical Society of America, 15, 1036-1047.

GeISLER, W. S., \& CHOU, K. L. (1995). Separation of low-level and highlevel factors in complex tasks: Visual search. Psychological Review, 102, 356-378.

GRAHAM, N. (1989). Visual pattern analyzers. New York: Oxford University Press.

Graham, N., Rico, M., Offen, S., \& Scott, W. (1999). Texture segregation shows only a very small lower-hemifield advantage. Vision Research, 39, 1171-1175.

Gurnsey, R., Pearson, P., \& DAy, D. (1996). Texture segmentation along the horizontal meridian: Nonmonotonic changes in performance with eccentricity. Journal of Experimental Psychology: Human Perception \& Performance, 22, 738-757.

He, S., Cavanagh, P., \& Intrilligator, J. (1996). Attentional resolution and the locus of visual awareness. Nature, 383, 334-337.

Hofmann, M. I., \& Hallett, P. E. (1993). Texture segregation based on 
two-dimensional relative phase differences in composite sine-wave grating patterns. Vision Research, 33, 221-234.

Joffe, K. M., \& Scialfa, C. T. (1995). Texture segmentation as a function of eccentricity, spatial frequency and target size. Spatial Vision, 9, 325-342.

JoNIDES, J. (1981). Voluntary vs. automatic control over the mind's eye's movement. In J. B. Long \& A. D. Baddeley (Eds.), Attention and performance IX (pp. 187-204). Hillsdale, NJ: Erlbaum.

JuLESZ, B. (1981). A theory of preattentive texture discrimination based on first-order statistics of textons. Biological Cybernetics, 41, 131138 .

KeHRER, L. (1989). Central performance drop on perceptual segregation tasks. Spatial Vision, 4, 45-62.

Lamme, V. A. F., Van Dijk, B. W., \& Spekreijse, J. (1993). Organization of texture segregation processing in primate visual cortex. Vision Neuroscience, 10, 781-790.

Lee, D. K., Itti, C., Косн, C., \& Braun, J. (1999). Attention activates winner-take-all competition among visual filters. Nature Neuroscience, 2, 375-381.

LeE, D. K., КосH, C., \& Braun, J. (1997). Spatial vision thresholds in the near absence of attention. Vision Research, 37, 2409-2418.

Lu, Z. L., \& Dosher, B. A. (1998). External noise distinguishes attention mechanisms. Vision Research, 38, 1183-1198.

Lu, Z L., Liu, C. Q., \& Dosher, B. (2000). Attention mechanisms for multilocation first- and second-order motion perception. Vision Research, 40, 173-186.

Luck, S. J., Hillyard, S. A., Mouloua, M., Woldorff, M. G., Clark, V. P., \& Hawkins, H. L. (1996). Effects of spatial cuing on luminance detectability: Psychophysical and electrophysiological evidence for early selection. Journal of Experimental Psychology: Human Perception \& Performance, 20, 887-904.

Mackeben, M. (1999). Sustained focal attention and peripheral letter recognition. SpatialAttention, 12, 51-72.

Mayfrank, L., Kimmig, H., \& Fischer, B. (1987). The role of attention in the preparation of visually guided saccadic eye movements in man. In J. K. O'Regan \& A. Levy-Schoen (Eds.), Eye movements: From physiology to cognition (pp. 37-45). New York: North-Holland.

Moran, J., \& Desimone, R. (1985). Selective attention gates visual processing in the extrastriate cortex. Science, 229, 782-784.

Morgan, M. J., Ward, R. M., \& CASTET, E. (1998). Visual search for a tilted target: Tests of spatial uncertainty models. Quarterly Journal of Experimental Psychology, 51A, 343-370.

MoriKaWA, K. (2000). Central performance drop in texture segmentation: The role of spatial and temporal factors. Vision Research, 40, 3455-3552.

Müller, M. M., Picton, T. W., Valdes-Sosa, P., Riera, J., TederSalejarvi, W. A., \& Hillyard, S. A. (1998). Effects of spatial attention on the steady-state visual evoked potential in the $20-28 \mathrm{~Hz}$ range. Cognitive Brain Research, 6, 249-261.

Nakayama, K., \& Mackeben, M. (1989). Sustained and transient components of focal visual attention. Vision Research, 29, 1631-1646.

NAZIR, T. A (1992). Effects of lateral masking and pre-cueing on gap resolution in central and peripheral vision. Vision Research, 32, 771-777.

NoRThdurft, H. C. (1991). Texture segmentation and pop-out from orientation contrast. Vision Research, 31, 1073-1078.

Palmer, J. (1994). Set-size effects in visual search: The effect of attention is independent of the stimulus for simple tasks. Vision Research, 34, 1703-1721.

Pearson, P., \& Gurnsey, R. (1992). Texture discrimination with foveal and peripheral presentation. Investigative Ophthalmology \& Visual Science, 33, 960.

Perry, V. H., \& Cowey, A. (1984). Retinal ganglion cells that project to the superior colliculus and pretectum in the macaque monkey. Neuroscience, 12, 1125-1137.

Perry, V. H., \& Cowey, A. (1985). The ganglion cell and cone distribution in the monkey retina: Implications for central magnification factors. Vision Research, 25, 1795-1810.

Posner, M. I. (1980). Orienting of attention. Quarterly Journal of Experimental Psychology, 32, 3-25.

Previc, F. H. (1990). Functional specialization in the lower and upper visual fields in humans: Its ecological origins and neurophysiological implications. Behavioral \& Brain Sciences, 13, 519-575.
Purpura, K. P., Victor, J. D., \& Katz, E. (1994). Striate cortex extracts higher-order spatial correlations from visual textures. Proceedings of the National Academy of Sciences, 91, 8482-8486.

Reynolds, J. H., \& Desimone, R. (1999). The role of neural mechanisms of attention in solving the binding problem. Neuron, 24, 19-29.

RijsdiJK, J. P., Kroon, J. N., \& VAN der Wilt, G. J. (1980). Contrast sensitivity as a function of position on the retina. Vision Research, $\mathbf{2 0}$, 235-241.

Rovamo, J., \& Virsu, V. (1979). An estimation and application of the human cortical magnification factor. Experimental Brain Research, 37, 495-510.

Rubin, N., Nakayama, K., \& Shapley, R. (1996). Enhanced perception of illusory contours in the lower versus upper visual hemifields. Science, 271, 651-653.

ShiU, L., \& PASHLER, J. (1994). Negligible effect of spatial precueing on identification of single digits. Journal of Experimental Psychology: Human Perception \& Performance, 20, 1037-1054.

Shiu, L., \& PAShler, J. (1995). Spatial attention and vernier acuity. Vision Research, 35, 337-343.

Solomon, J. A., Lavie, N., \& Morgan, M. J. (1997). Contrast discrimination function: Spatial cuing effects. Journal of the Optical Society of America, 14, 2443-2448.

Sperling, G., \& Dosher, B. A. (1986). Strategy and optimization in human information processing. In K. R. Boff, L. Kaufman, \& J. P. Thomas, Handbook of perception and human performance (Vol. 1, pp. 1-65). New York: Wiley.

Tootell, R. B. H., Swit kes, E., Silverman, M. S., \& Hamilton, S. L. (1998). Functional anatomy of macaque striate cortex, II: Retinotopic organization. Journal of Neuroscience, 8, 1531-1568.

Tsal, Y., \& Shalev, L. (1996). Inattention magnifies perceived length: The attentional receptive field hypothesis. Journal of Experimental Psychology: Human Perception \& Performance, 22, 233-243.

Van Essen, D. C., Newsome, W. T., \& Maunsell, J. H. R. (1984). The visual field representation in striate cortex of the macaque monkey: Asymmetries, anisotropies, and individual variability. Vision Research, 24, 429-448.

Verghese, P., \& Nakayama, K. (1994). Stimulus discriminability in visual search. Vision Research, 18, 2453-2467.

Yeshurun, Y., \& CARRAsco, M. (1998). Attention improves or impairs visual performance by enhancing spatial resolution. Nature, 396, 72-75.

Yeshurun, Y., \& CARRAsco, M. (1999). Spatial attention improves performance in spatial resolution tasks. Vision Research, 39, 293-305.

Yeshurun, Y., \& Carrasco, M. (2000). The locus of attentional effects in texture segmentation. Nature Neuroscience, 3, 622-627.

\section{NOTES}

1. A temporal explanation posits that the CPD stems from slower neural processing in central than in peripheral vision (Kehrer, 1989; Morikawa, 2000). This explanation rests on the fact that a mask follows a rapid display presentation, and its effect is greater at central than at peripheral locations. However, Yeshurun and Carrasco's (2000) finding that the CPD is present in the presence (Experiments 1 and 2) or absence (Experiments 3 and 4) of a mask indicates that the CPD does not require high temporal resolution.

2. A recent visual search study has shown that transient attention not only improves discriminability but also accelerates the rate of information processing (Carrasco \& McElree, 2001). However, in the texture task, the attentional impairment at the central locations cannot be explained by a temporal factor. Acceleration in the rate of processing should always improve performance.

3. Three trained psychophysical observers exhibited the same pattern of results as the naive observers.

4. Another study from our lab that was conducted subsequent to the present study and published recently (Carrasco et al., 2002) also shows (1) higher spatial resolution in an acuity task in the lower than in the upper VM; and (2) that transient attention affects acuity performance to a similar extent in both halves of the VM.

(Manuscript received June 20, 2001; revision accepted for publication October 23, 2001.) 